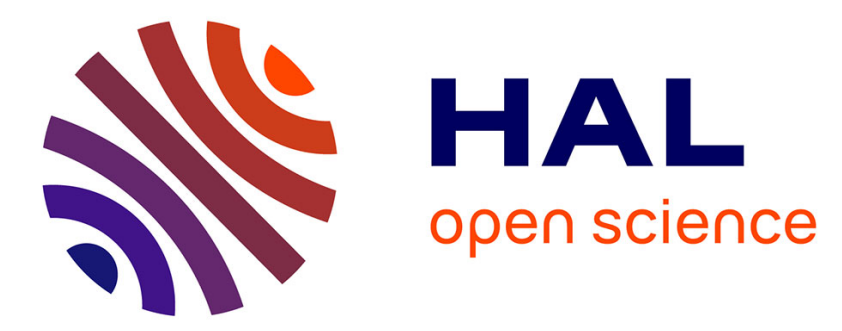

\title{
Spectrométrie neutronique pratique et approche à l'aide de détecteurs ionographiques visuels
}

M. Debeauvais, R. Stein, G. Rémy, J. Ralarosy, J. Tripier

\section{To cite this version:}

M. Debeauvais, R. Stein, G. Rémy, J. Ralarosy, J. Tripier. Spectrométrie neutronique pratique et approche à l'aide de détecteurs ionographiques visuels. Revue de Physique Appliquée, 1969, 4 (2), pp.259-260. 10.1051/rphysap:0196900402025900 . jpa-00243250

\section{HAL Id: jpa-00243250 https://hal.science/jpa-00243250}

Submitted on 1 Jan 1969

HAL is a multi-disciplinary open access archive for the deposit and dissemination of scientific research documents, whether they are published or not. The documents may come from teaching and research institutions in France or abroad, or from public or private research centers.
L'archive ouverte pluridisciplinaire HAL, est destinée au dépôt et à la diffusion de documents scientifiques de niveau recherche, publiés ou non, émanant des établissements d'enseignement et de recherche français ou étrangers, des laboratoires publics ou privés. 


\title{
SPEGTROMÉTRIE NEUTRONIQUE PRATIQUE ET APPROGHE A L'AIDE DE DÉTEGTEURS IONOGRAPHIQUES VISUELS
}

\author{
M. DEBEAUVAis, R. STEIN, G. RÉMY, J. RALAROSY et J. TRIPIER, \\ Centre de Recherches Nucléaires, Strasbourg-Cronenbourg.
}

\begin{abstract}
Résumé. - A l'aide de détecteurs solides visuels, on fait une spectrométrie neutronique entre 0 et $20 \mathrm{MeV}$. On utilise : $i$ ) entre 0 et $10 \mathrm{MeV}$, les réactions de fission sur diverses cibles : uranium naturel et enrichi avec et sans écran de cadmium, neptunium et thorium ; ii) entre 3 et $20 \mathrm{MeV}$, les réactions $(n, \alpha)$ sur cibles de béryllium, carbone et oxygène. Les détecteurs utilisés sont le Makrofol pour les réactions de fission et la nitrocellulose pour les réactions (n, $\alpha)$.
\end{abstract}

Abstract. - A spectrometer for 0 to $20 \mathrm{MeV}$ neutrons is made with the aid of visual solid detectors. The following reactions are used : i) fission reactions on natural and enriched uranium with and without cadmium, neptunium and thorium targets, for 0 to $10 \mathrm{MeV}$ neutrons ; ii) $(\mathrm{n}, \alpha)$ reactions on beryllium, carbon and oxygen targets, for 3 to $20 \mathrm{MeV}$ neutrons. Makrofol and nitrocellulose are respectively used as detectors for fission and $(n, \alpha)$ reactions.

La trace laissée par le passage de la particule ionisante, dans un haut polymère utilisé comme détecteur, peut être agrandie et visualisée par une attaque chimique appropriée [1]. Ghaque polymère possède un seuil de détection propre pour un développement standard [2]. Nous utilisons le Makrofol (polycarbonate), dont le seuil d'enregistrement se situe à 5,5 $\times 10^{3} \mathrm{MeV} . \mathrm{cm}^{2} / \mathrm{g}$, ainsi qu'un nitrate de cellulose de seuil correspondant à $1,3 \times 10^{3} \mathrm{MeV} . \mathrm{cm}^{2} / \mathrm{g}$. En spectrométrie neutronique, on se sert des réactions de fission sur cibles à seuils de fission différents et cela jusqu'aux environs de $3 \mathrm{MeV}$ (détecteur : Makrofol). Pour des énergies supérieures à $3 \mathrm{MeV}$, nous utilisons des réactions $(\mathrm{n}, \alpha)$ et, comme détecteur, de la nitrocellulose qui permet de visualiser des $\alpha$ d'énergies comprises entre 3,5 et $0,5 \mathrm{MeV}$.

Techniques expérimentales. - Les cibles utilisées sont l'uranium naturel, l'uranium enrichi, le neptunium et le thorium. Ces métaux sont évaporés directement sur le détecteur, ou utilisés en couche épaisse selon le flux à déterminer et la méthode de dépouillement utilisée [6]. Pour des énergies de neutrons supérieures à $3 \mathrm{MeV}$, on utilise la réaction ${ }^{9} \mathrm{Be}(\mathrm{n}, 2 \mathrm{n}) 2 \alpha$ en déposant Be sur le nitrate de cellulose et en observant les $2 \alpha$ en corrélation dans les deux feuilles de détecteur appliquées de part et d'autre de la cible. Au-delà de $10 \mathrm{MeV}$, la nitrocellulose sert en même temps de cible et de détecteur par la mise en évidence de la réaction ${ }^{12} \mathrm{G}\left(\mathrm{n}, \mathrm{n}^{\prime}\right) 3 \alpha$ induite sur les noyaux de carbone de l'édifice macromoléculaire lui-même.

Méthode de détermination des spectres. - Le dépouillement du nombre d'événements peut se faire par comptage individuel au microscope optique, ou par mesure globale (photométrie, différence de transmission de la lumière) [3]. Dans une première méthode de calcul, nous avons essayé de faire une résolution par bande d'énergie, mais ces résultats ne sont pas toujours satisfaisants, de sorte que nous avons dû faire une hypothèse sur l'allure du flux de neutrons en fonction de l'énergie. De nombreux auteurs [4] ont étudié les spectres neutroniques de fission ainsi que la déformation de ces mêmes spectres par des écrans de nature diverse. Tous ces auteurs admettent que le flux $\varphi(E)$ varie comme $1 / E$ dans le domaine d'énergie compris entre $0,1 \times 10^{-6}$ et $1,5 \mathrm{MeV}$ et varie exponentiellement entre 1,5 et $10 \mathrm{MeV}$. Pour la détermination des spectres, nous avons adopté des allures de flux pour les domaines d'énergie indiqués, mais nous avons introduit une forme de flux différente dans le cas des énergies inférieures à $0,1 \mathrm{eV}$, domaine que les auteurs précédemment cités traitent d'une façon à notre avis peu satisfaisante, en supposant le flux indépendant de l'énergie.

Les études deflux de neutrons thermiques ont montré que la distribution du nombre de neutrons en fonction de l'énergie correspondait à une fonction de Maxwell. Nous avons donc adopté cette allure de flux pour décrire la répartition des neutrons thermiques de fission, pour des énergies comprises entre 0 et $0,1 \mathrm{eV}$ (on a déterminé les conditions aux limites [4]).

Le nombre de bandes d'énergie dépend avant tout du nombre de cibles à seuil de fission différent que nous utilisons. Jusqu'à présent, nous avons pu opérer aisément sur les cibles suivantes : uranium naturel, uranium naturel sous écran de cadmium, neptunium, thorium et les réactions $(n, \alpha)$ déjà citées. Le tableau I donne un résumé des déterminations de flux déjà réalisées 


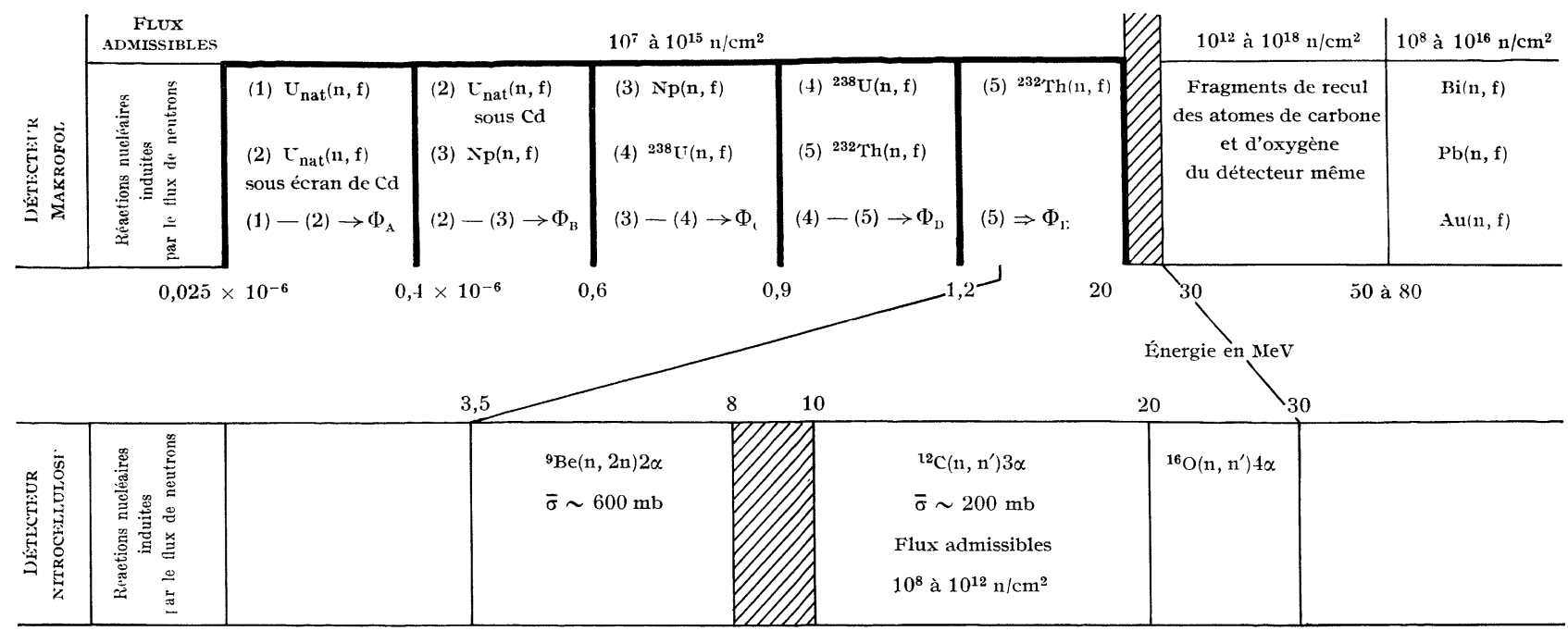

avec ces détecteurs. Lorsqu'on se sert des réactions $(n, \alpha)$ avec des détecteurs plus sensibles, le tableau I indique la méthode à utiliser en fonction des bandes d'énergie à étudier. Nous ne considérons que les réactions $(n, \alpha)$ donnant des événements caractéristiques (par exemple $\left.{ }^{9} \mathrm{Be}(\mathrm{n}, 2 \mathrm{n}) 2 \alpha,{ }^{12} \mathrm{G}\left(\mathrm{n}, \mathrm{n}^{\prime}\right) 3 \alpha,{ }^{16} \mathrm{O}\left(\mathrm{n}, \mathrm{n}^{\prime}\right) 4 \alpha\right)$. Une étude particulière [5] a été faite pour la réaction $\mathrm{G}\left(\mathrm{n}, \mathrm{n}^{\prime}\right) 3 \alpha$ où l'on a vérifié que, pour des neutrons de $14 \mathrm{MeV}$, le nombre de tripartitions était proportionnel, en coordonnées bilogarithmiques, au flux de neutrons. La section efficace de cette réaction étant sensiblement constante dans la bande d'énergie comprise entre 10 et $20 \mathrm{MeV}$, la détermination du nombre de neutrons est ainsi possible.
Les émulsions ionographiques permettent une spectrométrie fine de neutrons, mais, malgré toutes les méthodes de discrimination proposées, il reste difficile de faire des mesures lorsque le flux intégré dépasse $10^{9} \mathrm{n} / \mathrm{cm}^{2}$. C'est pourquoi la spectrométrie de neutrons basée sur les réactions de fission avec des cibles à seuils et des détecteurs insensibles aussi bien à la chaleur et aux déformations mécaniques (onde de choc) qu'aux particules $\beta, \gamma$, accompagnant les neutrons, reste seule utilisable sur le terrain. Ces détecteurs permettent d'attendre le temps nécessaire à une décontamination sans préjudice pour les mesures et sans nuire aux résultats; ce qui n'est pas le cas pour les méthodes d'analyse d'activation, dont l'exploitation doit être immédiate.

\section{BIBLIOGRAPHIE}

[1] Price et Walker, J. Appl. Phys., 1962, 33, 3407.

[2] Debenuvais (M.) et Monnin, C. R. Acad. Sc., 1965, 261, 2216.

[3] Debeauvais (M.), Coll. franco-allemand sur la mesure des rayonnements nucléaires, Regensburg, 1966.
[4] Frentz (C.), Diplôme d'Études Supérieures, Strasbourg, 1968.

[5] Mosinsky (G.), Thèse de $3^{\text {e }}$ cycle, Physique Corpusculaire, 1968.

[6] STEIN (R.) et al., Coll. intern. sur l'électronique nucléaire et la radioprotection, Toulouse, 1968. 